\title{
Placebo Effect of Sham Spine Procedures in Chronic Low Back Pain: A Systematic Review
}

\author{
Ammer M Jamjoom ' \\ Rothaina J Saeedi ${ }^{2}$ \\ Abdulhakim B Jamjoom (D) ${ }^{2}$ \\ 'Department of Trauma and \\ Orthopedics, Leeds General Infirmary, \\ Leeds, UK; ${ }^{2}$ Section of Neurosurgery, \\ King Khalid National Guards Hospital and \\ King Saud Bin Abdulaziz University for \\ Health Sciences, Jeddah, Saudi Arabia
}

Background: The literature lacks information about the characteristics of the placebo effect following sham spine procedures for chronic low back pain. We aim to evaluate the effect using pain score data from the sham arms of published trials.

Methods: Relevant trials were selected and reviewed. Baseline and post-procedure pain scores were collected. Each follow up pain score was considered an episode and compared to its baseline for significance. Patients and episodes were pooled and analyzed using three parameters: patient reported outcome measures (PROMs) (Oswestry Disability Index [ODI], Visual Analog Scale [VAS], Numerical Rating Scale [NRS] and Short Form-36 [SF]), anatomical targets (disc, facet, sacroiliac joint [SIJ], ramus communicans nerve [RCN], basivertebral nerve [BVN], and caudal) and follow up periods (early: 0-2, intermediate: $>2-4$ and late: $>4-6$ ) in months. The percentage of pooled patients in the episodes that had significant reduction in pain scores was termed placebo effect. The outcome was defining the magnitude of the placebo effect and determining if it was influenced by the three parameters. Results: Seventeen studies that reported 535 patients and 55 pain scoring episodes were considered eligible. Significant reduction in pain scores was reported in 21 episodes. The overall placebo effect among the patients during the studied period was $53.2 \%$. The rate ranged according to PROMs from $42.4 \%$ to $72.1 \%$, anatomical targets from $11.1 \%$ to $100 \%$ and follow up periods from $47.9 \%$ to $59 \%$. The placebo effect differed significantly between the various domains in the three parameters.

Conclusion: Placebo effect was observed in nearly half of the patients during the first 6 months following a sham spine procedure. The effect was influenced by utilized PROMs, anatomical target and follow up period. The findings should be considered in the design of new sham spine procedure trials. Further research is required to delineate the effect of bias on the findings.

Keywords: sham procedure, sham surgery, placebo effect, spine, trials

\section{Introduction}

Sham surgical trials have been controversial. Several authors considered them unsafe as they may subject patients to the unwarranted risks of invasive procedures. Others believed sham surgical trials were ethical and appropriate where there is poor evidence on the efficacy of the intervention. ${ }^{1,2}$ In placebo-controlled surgical research, surgery is defined as an invasive intervention that entails access to the body via an incision, natural orifice, or percutaneous puncture, which includes the use of instrumentation and requires operator skill. ${ }^{3}$

The placebo effect of sham surgical procedures has been a matter of interest in recent years. $^{1,2,4,5}$ It is defined as the clinical or behavioral improvement following

\footnotetext{
Correspondence: Abdulhakim B Jamjoom King Khalid National Guards Hospital P.O. Box 9515, Jeddah, 21423 , Saudi Arabia

Tel +966 I2 2266666 (Ext. 2207I)

Email jamjoomab@gmail.com
} 
placebo manipulation or sham intervention. ${ }^{6}$ It is accepted that the placebo response describes the difference between the baseline and follow up measures while the placebo effect refers to the changes in response associated with treatment, conditioning, and expectation. ${ }^{4}$ In this article the term placebo effect was used to imply the placebo effect and response as the two terms are often used interchangeably. ${ }^{4}$ It is recognized that the placebo effect in the context of surgical care could be substantial in magnitude and may remain significant throughout the blinded follow up. ${ }^{2,4}$ The effect can be associated with a large improvement in pain and other subjective measures but with less effect on objective outcomes. ${ }^{1}$ The effect might be influenced by the way patients are informed about the placebo control, ${ }^{2}$ and by certain trial characteristics such the number of subjects, the frequency of face-to -face visits, subject baseline pain intensity, study design, and location. ${ }^{4}$

At present the literature lacks studies that focused on the characteristics of the placebo effect following sham spine procedures. Information relating to the features of the placebo effect and the factors influencing it will be useful in the design and interpretation of future spine procedure placebo-controlled trials. The purpose of this study is to define the placebo effect following sham spine procedures using data from the sham arm of published randomized controlled trials (RCTs). We also aim to ascertain whether the placebo effect was influenced by the utilized patient reported outcome measures (PROMs), the anatomical targets for the sham procedure or the duration of the follow up.

\section{Methods}

\section{Eligibility Criteria}

We included all RCTs that reported patients who underwent sham spine procedures as the control arm of a treatment modality for chronic low back pain in the absence of radiculopathy. Sham procedure was defined as a therapeutic intervention in which there was a breach in the skin and the patient received either nothing, or an injection of saline or local anesthesia (LA). It is accepted that the use of LA may influence pain scores in the immediate post-procedure period. However, as the effect is usually limited to few hours, it was deemed appropriate to consider the injection of LA as a sham procedure. The inclusion criteria were RCTs published in the English language from 1999 to 2019, that reported a minimal of 10 patients and recorded a baseline and at least one follow-up pain score. The PROMs were restricted to the four most used instruments which were: Oswestry Disability Index (ODI), Visual Analog Scale (VAS), Numerical Rating Scale (NRS) and Short Form (SF)-36. Scores from SF-36 were limited to the pain domain. The exclusion criteria are illustrated in Table 1.

\section{Literature Search}

The literature was systematically searched in November 2020 using a combination or part combination of the following terms: sham, surgery, procedure, spine, back pain, and trials. Two authors independently interrogated the literature using the databases PubMed, Medline, and the Cochrane Central Register of Controlled Trials. The full texts of potentially appropriate studies were retrieved and assessed. The following data were collected for each study: year of publication, number of patients in the sham arm, the PROMs instrument used, all recorded baseline and follow up pain scores, anatomical target for the procedure, duration of the sham procedure, use of LA, treated versus sham group pain scores and follow up duration. Missing data were referred to as not available (NA).

Table I Sham Spine Procedures in Low Back Pain Selection Flow Diagram

\begin{tabular}{l}
$\begin{array}{l}\text { Search and Number of Studies: } \\
\text { - Sham Surgery AND Back Pain OR Spine AND Trial = I27 } \\
\text { - Sham Procedure AND Back Pain OR Spine AND Trial = } 310\end{array}$ \\
\hline \multicolumn{1}{c|}{ Total screened studies = 437 } \\
\hline Studies excluded after title and abstract screening = 376 \\
\hline Remaining studies = 6I \\
The following studies were excluded after full articles assessment for \\
eligibility = Non-invasive treatment (I5) \\
- No sham group (7) \\
- Review articles (6) \\
- Vertebroplasty (4) \\
- Focus on diagnosis not therapy (3) \\
- Radiculopathy (3) \\
- Incomplete pain scores (2) \\
- Sham group less than I0 patients (2) \\
- Sham group received steroids (I) \\
- Publication before I999 (I) \\
Remaining studies = I7 \\
Studies eligible for the review = I7 \\
\hline
\end{tabular}




\section{Data Analysis}

Variation in the timing of the follow up pain scores necessitated grouping the results into three periods in months: early from $0-2$, intermediate: $>2-4$ and late: $>4-6$. Each follow pain score was referred to as an episode. The number of possible episodes in a trial ranged from 1 to 12 (3 periods $\times 4$ PROMs). Each pain scoring episode was compared with its baseline and examined statistically. The total patients and episodes were pooled and analyzed based on the following three parameters: 1) four PROMs instruments (ODI, VAS, NRS, SF-36), 2) six anatomical targets of the sham procedures (disc, facet, sacroiliac joint [SIJ], ramus communicans nerve [RCN], basivertebral nerve [BVN], caudal), and 3) three follow up periods (early, intermediate, late).

\section{Outcome Measures}

The outcome was defining the placebo effect and determining whether it was influenced by three parameters (PROMs, anatomical targets and follow up periods).

\section{Assessment of Methodological Quality}

Two of the authors assessed the methodological quality of the articles independently using the modified Jadad quality scale. Each study was scored as follows: randomization (2-0), blinding (2-0), withdrawals and dropouts (1-0), inclusion and exclusion criteria (1-0), adverse effects $(1-0)$ and the statistical analysis (1-0). A total score (0 to 8$)$ was calculated. This score was used to define the perceived risk of bias which was considered low for scores $\geq 7$, intermediate for scores 6 5 and high for scores $\leq 4$.

\section{Statistical Analysis}

The baseline and follow up scores in each episode were evaluated statistically using the mean difference (MD) test from an online source. ${ }^{7}$ The placebo effect was defined by calculating the percentage of pooled patients in the episodes that had significant reduction in pain scores compared to the patients in all the episodes. The influence of the three parameters (PROMs, anatomical targets and follow up periods) on the placebo effect was assessed by comparing the distribution of the pooled number of patients that had significant and non-significant reduction in pain scores between the various domains in each of the three parameters. The comparison was carried out using a chi-squared test from an online source. ${ }^{8}$ In all the statistical analyses, significance was achieved at $\mathrm{P}<0.05$.

\section{Results}

Study Selection, Characteristics, and

\section{Quality}

The literature search yielded 17 sham-controlled trials of spine procedures for chronic low back pain that were suitable. $^{9-25}$ The characteristics of patients are summarized in Table 2. The 17 articles reported a total of 535 patients and the number of patients per article was 10-77 (median 30). The duration of the sham spine procedure was 1-16.5 (median 1.5) minutes. The 17 studies recorded a total of 55 pain scoring follow up episodes. The number of episodes per study was 1-6 (median 3.5) (Table 3). As some of the 535 patients were evaluated using more than one PROM and at more than one follow up period, the 55 episodes reflected experience equivalent to a total of 1641 patients. The total numbers of patients (and episodes) based on PROMs, anatomical targets and follow up periods were: ODI: 703 (23), VAS: 389 (12), NRS: 398 (13), SF-36: 151 (7), disc: 542 (23), facet: 287 (10), BVN: 308 (4), RCN: 104 (5), SIJ: 160 (9), caudal: 240 (4), early: 337 (14), intermediate: 684 (20) and late: 656 (21) (Table 4).

The overall quality of articles was considered intermediate to high with the mean (range) Jadad score $6.6(6-8)$. The bias risk was considered intermediate in 5 studies, ${ }^{10,11,16,19,20}$ and low in the remaining 12 articles (Table 2).

\section{Study Findings}

Table 3 summarizes the baseline, follow up pain scores and MD statistical analysis for the 55 pain scoring episodes based on the three parameters (PROMs, anatomical targets and follow up periods). Table 4 shows the distribution of the pooled number of patients and episodes that had significant and non-significant reduction in pain scores between the various domains of each of the three parameters.

The overall number of episodes that had significant reduction in pain scores was 21 out of 55 . The number of episodes that had significant reduction in pain scores compared to the total number of episodes based on the various domains were: ODI: $7 / 23$, VAS: $4 / 12$, NRS: $8 / 13$, SF-36: $2 /$ 7, disc: $2 / 23$, facet: $6 / 10$, BVN: $4 / 4$, RCN: $3 / 5$, SIJ: $2 / 9$, caudal: $4 / 4$, early: $7 / 14$, intermediate: $8 / 20$ and late: $6 / 21$.

The overall placebo effect among all the patients was 873/1641 (53.2\%). The placebo effect based on the various domains was: ODI: $46.7 \%$, VAS: $49.9 \%$, NRS: $72.1 \%$, SF36: $42.4 \%$, disc: $11.1 \%$, facet: $52.6 \%$, BVN: $100 \%$, RCN: $64.4 \%$, SIJ: $29.4 \%$, caudal: $100 \%$, early: $52.5 \%$, 
Table 2 The Characteristics of the Sham Spine Procedure Patients in the 17 Selected RCTs

\begin{tabular}{|c|c|c|c|c|c|c|c|}
\hline $\begin{array}{l}\text { Author }{ }^{\text {ref }} \\
\text { Year }\end{array}$ & $\begin{array}{l}\text { Cases } \\
\text { No. }\end{array}$ & $\begin{array}{l}\text { Anatomical Target for the } \\
\text { Sham Procedure }\end{array}$ & $\begin{array}{l}\text { Sham } \\
\text { Dur. }\end{array}$ & LA Given & Treat. & $\begin{array}{l}\text { Pain Score Findings Treated vs } \\
\text { Sham Groups }\end{array}$ & $\begin{array}{l}\text { Bias } \\
\text { Risk }\end{array}$ \\
\hline Laclaire $^{9} 200 \mathrm{I}$ & 34 & $\begin{array}{l}\text { Facet: Proximal and distal } \\
\text { nerve }\end{array}$ & $90 \mathrm{sec}$ & $\begin{array}{l}\text { Lidocaine } \\
1 \%(2 \mathrm{mls})\end{array}$ & RFA & Comparable ODI, VAS (4, 12 weeks) & Low \\
\hline Pauza $^{10} 2004$ & 24 & Disc & $\begin{array}{l}16.5 \\
\min \end{array}$ & None & IDET & $\begin{array}{l}\text { Comparable VAS \& SF- } 36 \text { Better } \\
\text { ODI in treated group ( } 6 \text { months) }\end{array}$ & Interm. \\
\hline$O h^{\prime \prime} 2004$ & 23 & $\begin{array}{l}\text { RCN: Lateral portion of } \\
\text { vertebral body }\end{array}$ & $60 \mathrm{sec}$ & $\begin{array}{l}\text { Lignocaine } \\
\mid \%(2 \mathrm{mls})\end{array}$ & RFA & $\begin{array}{l}\text { Better VAS \& SF- } 36 \text { in treated group } \\
\text { (4 months) }\end{array}$ & Interm. \\
\hline $\begin{array}{l}\text { Freeman } \\
2005\end{array}$ & 19 & Disc & $\begin{array}{l}16.5 \\
\min \end{array}$ & None & IDET & $\begin{array}{l}\text { Comparable ODI \& SF-36 (6 } \\
\text { months) }\end{array}$ & Low \\
\hline $\begin{array}{l}\text { Van Wijik }{ }^{13} \\
2005\end{array}$ & 41 & $\begin{array}{l}\text { Facet: Superior articular and } \\
\text { transverse processes }\end{array}$ & $60 \mathrm{sec}$ & $\begin{array}{l}\text { Mepivacaine } \\
2 \%(0.5 \mathrm{mls})\end{array}$ & RFA & Comparable SF-36 (3 months) & Low \\
\hline Tekin $^{14} 2007$ & 20 & $\begin{array}{l}\text { Facet: Superior articular and } \\
\text { transverse processes }\end{array}$ & $90 \mathrm{sec}$ & $\begin{array}{l}\text { Bupivacaine } \\
0.5 \% \\
(0.3 \mathrm{mls})\end{array}$ & RFA & $\begin{array}{l}\text { Better ODI \& VAS in treated group } \\
\text { (post-procedure and } 6 \text { months) }\end{array}$ & Low \\
\hline Cohen ${ }^{15} 2008$ & 14 & $\begin{array}{l}\text { SIJ: L5 superior articular and } \\
\text { transverse processes, sacral } \\
\text { ala and SI-S3 foramina }\end{array}$ & NA & $\begin{array}{l}\text { Lidocaine } \\
2 \%(0.5 \mathrm{mls})\end{array}$ & RFA & $\begin{array}{l}\text { Better ODI \& NRS in treated group } \\
\text { ( } 1 \text { and } 3 \text { months) }\end{array}$ & Low \\
\hline $\begin{array}{l}\text { Kvarstein }^{16} \\
2009\end{array}$ & 10 & Disc & $10 \mathrm{~min}$ & $\begin{array}{l}\text { Bupivacaine } \\
0.5 \%(2 \mathrm{mls})\end{array}$ & RFA & $\begin{array}{l}\text { Comparable ODI \& SF-36 (6 } \\
\text { months) }\end{array}$ & Interm. \\
\hline Peng ${ }^{17} 2010$ & 36 & Disc & NA & $\begin{array}{l}\text { Lidocaine } \\
2 \%(I \mathrm{~mL})\end{array}$ & Inject & $\begin{array}{l}\text { Better ODI \& NRS in the treated } \\
\text { group ( } 6 \text { months) }\end{array}$ & Low \\
\hline $\mathrm{CaO}^{18} 20 \mathrm{II}$ & $\begin{array}{l}\text { A: } 20 \\
\text { B: } 20\end{array}$ & Disc & NA & None & Inject & $\begin{array}{l}\text { Better ODI \& VAS in the treated } \\
\text { group ( } 3 \text { and } 6 \text { months) }\end{array}$ & Low \\
\hline Patel $^{19} 2012$ & 17 & $\begin{array}{l}\text { SIJ: L5 superior articular and } \\
\text { transverse processes, sacral } \\
\text { ala and SI-S3 foramina }\end{array}$ & $\begin{array}{l}150 \\
\sec \end{array}$ & $\begin{array}{l}\text { Lidocaine } \\
2 \%(0.5 \mathrm{mls})\end{array}$ & RFA & $\begin{array}{l}\text { Better ODI \& SF- } 36 \text { in the treated } \\
\text { group ( } \mathrm{l} \text { and } 3 \text { months). Better NRS } \\
\text { ( } 3 \text { months) }\end{array}$ & Interm \\
\hline $\begin{array}{l}\text { Manchikanti }{ }^{20} \\
2012\end{array}$ & 60 & Caudal: Epidural space & NA & $\begin{array}{l}\text { Lidocaine } \\
0.5 \%(10 \mathrm{mls})\end{array}$ & Inject & Better ODI \& NRS ( 3 and 6 months) & Interm \\
\hline $\begin{array}{l}\text { Kapural }^{21} \\
2013\end{array}$ & 30 & Disc & $15 \mathrm{~min}$ & None & RFA & $\begin{array}{l}\text { Comparable ODI \& NRS ( } 1 \text { and } 3 \\
\text { months). Better ODI \& NRS in the } \\
\text { treated group ( } 6 \text { months) }\end{array}$ & Low \\
\hline $\begin{array}{l}\text { Van Tilburg } \\
2016\end{array}$ & 30 & $\begin{array}{l}\text { Facet: Superior articular and } \\
\text { transverse processes }\end{array}$ & $60 \mathrm{sec}$ & $\begin{array}{l}\text { Lidocaine } \\
2 \%(I \mathrm{~mL})\end{array}$ & RFA & Comparable NRS (I month) & Low \\
\hline $\begin{array}{l}\text { Van Tibugh } \\
2016\end{array}$ & 30 & $\begin{array}{l}\text { SIJ: Lateral, inferior border of } \\
\text { sacrum and I } \mathrm{cm} \text { lateral and } \\
\text { below } \mathrm{S} 4 \text { foramen }\end{array}$ & $90 \mathrm{sec}$ & $\begin{array}{l}\text { Lidocaine } \\
2 \%](10 \mathrm{mls})\end{array}$ & RFA & Comparable NRS (I month) & Low \\
\hline $\begin{array}{l}\text { Van Tiburg } \\
2017\end{array}$ & 30 & $\begin{array}{l}\mathrm{RCN} \text { : Caudal to transverse } \\
\text { process medial to lateral } \\
\text { border of vertebra }\end{array}$ & $60 \mathrm{sec}$ & $\begin{array}{l}\text { Lidocaine } \\
2 \%(I \mathrm{~mL})\end{array}$ & RFA & Comparable NRS (I month) & Low \\
\hline $\begin{array}{l}\text { Fishgrund }{ }^{25} \\
2018\end{array}$ & 77 & BVN: I-2 mm into pedicle & $15 \mathrm{~min}$ & None & RFA & $\begin{array}{l}\text { Better ODI in the treated group ( } 3 \\
\text { months) and VAS ( } 6 \text { months) }\end{array}$ & Low \\
\hline
\end{tabular}

Abbreviations: No, number; Dur, duration; LA, local anesthesia; Treat, treatment; vs, versus; Interm, intermediate; min, minutes; sec, seconds; ODI, Oswestry Disability Index; VAS, Visual Analog Scale; NRS, Numerical Rating Scale; SF-36, Short Form -36; RFA, Radiofrequency Ablation; IDET, Intradiscal Electrothermal Therapy; Inject, injection; BVN, basivertebral nerve; RCN, Ramus Communicans Nerve; SIJ, sacroiliac joint. 
Table 3 The Mean Difference Statistical Analysis of Baseline and Follow Up Mean Pain Scores in the Recorded 55 Episodes from 17 Studies Based on the PROMs, Anatomical Targets, and Follow Up Periods

\begin{tabular}{|c|c|c|c|c|c|c|}
\hline Author ref & $\begin{array}{l}\text { Anatomic } \\
\text { Target }\end{array}$ & $\begin{array}{l}\text { FU } \\
\text { Period }\end{array}$ & $\begin{array}{l}\text { PROMS } \\
\text { Instr. }\end{array}$ & $\begin{array}{l}\text { Mean Baseline Score } \\
( \pm S D)\end{array}$ & $\begin{array}{l}\text { Mean Sham FU Score } \\
( \pm S D)\end{array}$ & MD (P-value) \\
\hline \multirow[t]{2}{*}{ Laclaire ${ }^{9}$} & \multirow[t]{2}{*}{ Facet } & Early & $\begin{array}{l}\text { ODI } \\
\text { VAS }\end{array}$ & $\begin{array}{l}36.4( \pm 14.6) \\
5.15( \pm 2.08)\end{array}$ & $\begin{array}{l}34.4( \pm 9.4) \\
5.21( \pm 2.36)\end{array}$ & $\begin{array}{l}2(P=0.504) \\
-0.06(P=0.912)\end{array}$ \\
\hline & & Interm & $\begin{array}{l}\text { ODI } \\
\text { VAS }\end{array}$ & $\begin{array}{l}36.4( \pm 14.6) \\
5.15( \pm 2.08)\end{array}$ & $\begin{array}{l}33.7( \pm 9.1) \\
4.44( \pm 2.73)\end{array}$ & $\begin{array}{l}2.7(\mathrm{P}=0.364) \\
0.7(\mathrm{P}=0.232)\end{array}$ \\
\hline $\operatorname{Pauza}^{10}$ & Disc & Late & $\begin{array}{l}\text { ODI } \\
\text { VAS } \\
\text { SF-36 }\end{array}$ & $\begin{array}{l}33( \pm 11) \\
6.5( \pm 1.9) \\
35( \pm 12)\end{array}$ & $\begin{array}{l}28( \pm 15) \\
5.4( \pm 2.7) \\
44( \pm 20)\end{array}$ & $\begin{array}{l}5(P=0.194) \\
I . I(P=0.1 I) \\
-9(P=0.065)\end{array}$ \\
\hline$O h^{11}$ & $\mathrm{RCN}$ & Interm & $\begin{array}{l}\text { VAS } \\
\text { SF-36 }\end{array}$ & $\begin{array}{l}7( \pm 1.6) \\
28.8( \pm 3.6)\end{array}$ & $\begin{array}{l}6.3( \pm 1.1) \\
32.4( \pm 5.1)\end{array}$ & $\begin{array}{l}0.7(P=0.091) \\
-3.6(P=0.008)\end{array}$ \\
\hline Freeman $^{12}$ & Disc & Late & $\begin{array}{l}\text { ODI } \\
\text { SF-36 }\end{array}$ & $\begin{array}{l}40.74( \pm I I .84) \\
24.42(I 3.45)\end{array}$ & $\begin{array}{l}41.58( \pm 11.29) \\
31.47( \pm 15.29)\end{array}$ & $\begin{array}{l}-0.84(P=0.824) \\
-7.05(P=0.14)\end{array}$ \\
\hline Van Wijik ${ }^{13}$ & Facet & Interm & SF-36 & $31.2( \pm 15.3)$ & $42.8( \pm 20.6)$ & $-11.6(P=0.005)$ \\
\hline \multirow[t]{2}{*}{ Tekin $^{14}$} & \multirow[t]{2}{*}{ Facet } & Early & $\begin{array}{l}\text { ODI } \\
\text { VAS }\end{array}$ & $\begin{array}{l}40.1( \pm 2.8) \\
6.8( \pm 1.6)\end{array}$ & $\begin{array}{l}30.5( \pm 5.7) \\
4.3( \pm 1)\end{array}$ & $\begin{array}{l}9.6(\mathrm{P}<0.00 \mathrm{I}) \\
2.5(\mathrm{P}<0.00 \mathrm{I})\end{array}$ \\
\hline & & Late & $\begin{array}{l}\text { ODI } \\
\text { VAS }\end{array}$ & $\begin{array}{l}40.1( \pm 2.8) \\
6.8( \pm 1.6)\end{array}$ & $\begin{array}{l}28.9( \pm 5.7) \\
3.1( \pm 0.8)\end{array}$ & $\begin{array}{l}1 \mathrm{I} .2(\mathrm{P}<0.00 \mathrm{I}) \\
3.7(\mathrm{P}<0.00 \mathrm{I})\end{array}$ \\
\hline \multirow[t]{2}{*}{ Cohen $^{15}$} & \multirow[t]{2}{*}{ SIJ } & Early & $\begin{array}{l}\text { ODI } \\
\text { NRS }\end{array}$ & $\begin{array}{l}47.9( \pm 9.3) \\
6.5( \pm 1.9)\end{array}$ & $\begin{array}{l}43.6( \pm 14) \\
6.3( \pm 2.40)\end{array}$ & $\begin{array}{l}4.3(P=0.347) \\
0.2(P=0.809)\end{array}$ \\
\hline & & Interm & $\begin{array}{l}\text { ODI } \\
\text { NRS }\end{array}$ & $\begin{array}{l}47.9( \pm 9.3) \\
6.5( \pm 1.9)\end{array}$ & $\begin{array}{l}24( \pm 8.5) \\
6( \pm 2.4)\end{array}$ & $\begin{array}{l}23.9(P<0.00 I) \\
0.5(P=0.546)\end{array}$ \\
\hline Kvarstein $^{16}$ & Disc & Late & $\begin{array}{l}\text { ODI } \\
\text { SF-36 }\end{array}$ & $\begin{array}{l}30.4( \pm 15.3) \\
32.5( \pm 14.8)\end{array}$ & $\begin{array}{l}28.2( \pm 13.1) \\
35.3( \pm 18.6)\end{array}$ & $\begin{array}{l}2.2(P=0.734) \\
-2.8(P=0.7 \mid 4)\end{array}$ \\
\hline Peng ${ }^{17}$ & Disc & Late & $\begin{array}{l}\text { ODI } \\
\text { NRS }\end{array}$ & $\begin{array}{l}49.37( \pm 6.79) \\
6.73( \pm 1.15)\end{array}$ & $\begin{array}{l}48.4( \pm 7.77) \\
6.35( \pm 1.17)\end{array}$ & $\begin{array}{l}0.97(\mathrm{P}=0.575) \\
0.38(\mathrm{P}=0.169)\end{array}$ \\
\hline \multirow[t]{2}{*}{$\mathrm{Cao}^{18}$ (Group A) } & \multirow[t]{2}{*}{ Disc } & Interm & $\begin{array}{l}\text { ODI } \\
\text { VAS }\end{array}$ & $\begin{array}{l}37.9( \pm 14.65) \\
7.1( \pm 1.61)\end{array}$ & $\begin{array}{l}42( \pm 13.92) \\
7( \pm 1.33)\end{array}$ & $\begin{array}{l}-4.1 \quad(P=0.37) \\
0.1 \quad(P=832)\end{array}$ \\
\hline & & Late & $\begin{array}{l}\text { ODI } \\
\text { VAS }\end{array}$ & $\begin{array}{l}37.9( \pm 14.65) \\
7.1( \pm I .6 I)\end{array}$ & $\begin{array}{l}44.4( \pm 13.98) \\
7.5( \pm 1.08)\end{array}$ & $\begin{array}{l}-6.5 P=0.159) \\
-0.4(P=0.362)\end{array}$ \\
\hline \multirow[t]{2}{*}{$\mathrm{Cao}^{18}$ (Group B) } & \multirow[t]{2}{*}{ Disc } & Interm & $\begin{array}{l}\text { ODI } \\
\text { VAS }\end{array}$ & $\begin{array}{l}32.4( \pm 9.65) \\
6.5( \pm 1.2)\end{array}$ & $\begin{array}{l}33.3( \pm 10.63) \\
6.8( \pm 1.03)\end{array}$ & $\begin{array}{l}-0.9(P=0.781) \\
-0.3(P=0.402)\end{array}$ \\
\hline & & Late & $\begin{array}{l}\text { ODI } \\
\text { VAS }\end{array}$ & $\begin{array}{l}32.4( \pm 9.65) \\
6.5( \pm 1.2)\end{array}$ & $\begin{array}{l}33.8( \pm 11.95) \\
6.4( \pm 1.07)\end{array}$ & $\begin{array}{l}-1.4(P=0.686) \\
0.1 \quad(P=0.782)\end{array}$ \\
\hline \multirow[t]{2}{*}{ Patel $^{19}$} & \multirow[t]{2}{*}{ SIJ } & Early & $\begin{array}{l}\text { ODI } \\
\text { NRS } \\
\text { SF-36 }\end{array}$ & $\begin{array}{l}35( \pm 10) \\
5.8( \pm 1.3) \\
43( \pm 10)\end{array}$ & $\begin{array}{l}3 I( \pm I I) \\
4 . I( \pm 2.0) \\
45( \pm I I)\end{array}$ & $\begin{array}{l}4(P=0.276) \\
1.7(P=0.006) \\
2(P=0.595)\end{array}$ \\
\hline & & Interm & $\begin{array}{l}\text { ODI } \\
\text { NRS } \\
\text { SF-36 }\end{array}$ & $\begin{array}{l}35( \pm 10) \\
5.8( \pm 1.3) \\
43( \pm 10)\end{array}$ & $\begin{array}{l}37( \pm 6) \\
5( \pm 2.4) \\
42( \pm 13)\end{array}$ & $\begin{array}{l}-2(P=0.485) \\
0.8(P=0.236) \\
I(P=0.809)\end{array}$ \\
\hline
\end{tabular}


Table 3 (Continued).

\begin{tabular}{|c|c|c|c|c|c|c|}
\hline Author ref & $\begin{array}{l}\text { Anatomic } \\
\text { Target }\end{array}$ & $\begin{array}{l}\text { FU } \\
\text { Period }\end{array}$ & $\begin{array}{l}\text { PROMS } \\
\text { Instr. }\end{array}$ & $\begin{array}{l}\text { Mean Baseline Score } \\
( \pm S D)\end{array}$ & $\begin{array}{l}\text { Mean Sham FU Score } \\
( \pm S D)\end{array}$ & MD (P-value) \\
\hline \multirow[t]{2}{*}{ Manchikanti ${ }^{20}$} & \multirow[t]{2}{*}{ Caudal } & Interm & $\begin{array}{l}\text { ODI } \\
\text { NRS }\end{array}$ & $\begin{array}{l}28.3( \pm 4.92) \\
8( \pm 0.9)\end{array}$ & $\begin{array}{l}16.3( \pm 7.2) \\
4.2( \pm 1.8)\end{array}$ & $\begin{array}{l}12(P<0.001) \\
3.8(P<0.001)\end{array}$ \\
\hline & & Late & $\begin{array}{l}\text { ODI } \\
\text { NRS }\end{array}$ & $\begin{array}{l}28.3( \pm 4.92) \\
8( \pm 0.9)\end{array}$ & $\begin{array}{l}16.4( \pm 7.4) \\
4.1( \pm 1.8)\end{array}$ & $\begin{array}{l}11.9(P<0.001) \\
3.9(P<0.001)\end{array}$ \\
\hline \multirow[t]{3}{*}{ Kapural $^{21}$} & \multirow[t]{3}{*}{ Disc } & Early & $\begin{array}{l}\text { ODI } \\
\text { NRS }\end{array}$ & $\begin{array}{l}40.93( \pm 13.56) \\
7.18( \pm 1.98)\end{array}$ & $\begin{array}{l}39.85( \pm 17.03) \\
5.72( \pm 2.29)\end{array}$ & $\begin{array}{l}1.08(P=0.787) \\
\mathrm{I} .46(P=0.012)\end{array}$ \\
\hline & & Interm & $\begin{array}{l}\text { ODI } \\
\text { NRS }\end{array}$ & $\begin{array}{l}40.93( \pm 13.56) \\
7.18( \pm 1.98)\end{array}$ & $\begin{array}{l}40.44( \pm 16.2 I) \\
5.98( \pm 2.36)\end{array}$ & $\begin{array}{l}0.49(P=0.9) \\
I .2(P=0.04 \mathrm{I})\end{array}$ \\
\hline & & Late & $\begin{array}{l}\text { ODI } \\
\text { NRS }\end{array}$ & $\begin{array}{l}40.93( \pm 13.56) \\
7.18( \pm 1.98)\end{array}$ & $\begin{array}{l}41.17( \pm 13.94) \\
6.58( \pm 2.11)\end{array}$ & $\begin{array}{l}-0.24(P=0.946) \\
0.6(P=0.268)\end{array}$ \\
\hline Van Tilburg 22 & Facet & Early & NRS & $7.4( \pm 0.8)$ & $5.5( \pm 1.9)$ & $1.9(P=0.005)$ \\
\hline Van Tibugh ${ }^{23}$ & SIJ & Early & NRS & $7.5( \pm 1.2)$ & $5.4( \pm 1.9)$ & $2.1(P<0.00 I)$ \\
\hline Van Tiburg ${ }^{24}$ & $\mathrm{RCN}$ & Early & NRS & $7.8( \pm 1.05)$ & $5.7( \pm 2.28)$ & $2.1(P<0.001)$ \\
\hline \multirow[t]{2}{*}{ Fishgrund $d^{25}$} & \multirow[t]{2}{*}{ BVN } & Interm & $\begin{array}{l}\text { ODI } \\
\text { VAS }\end{array}$ & $\begin{array}{l}41.2( \pm 10.38) \\
6.64( \pm 1.34)\end{array}$ & $\begin{array}{l}25.8( \pm 17.44) \\
4.14( \pm 2.64)\end{array}$ & $\begin{array}{l}\text { I5.4 (P<0.00I }) \\
2.5(P<0.001)\end{array}$ \\
\hline & & Late & $\begin{array}{l}\text { ODI } \\
\text { VAS }\end{array}$ & $\begin{array}{l}41.2( \pm 10.38) \\
6.64( \pm 1.34)\end{array}$ & $\begin{array}{l}25.1( \pm 15.29) \\
4.41( \pm 2.76)\end{array}$ & $\begin{array}{l}16.1 \quad(P<0.001) \\
2.23(P<0.001)\end{array}$ \\
\hline
\end{tabular}

Notes: Early, 0-2 months; intermediate, >2-4 months; late, >4-6 months.

Abbreviations: No, number; FU, follow up; Instr, instrument; RROMS, patient reported outcome measures; MD, mean difference; SD, standard deviation; Interm, intermediate; BVN, basivertebral nerve; RCN, Ramus Communicans Nerve; SIJ, sacroiliac joint; ODI, Oswestry Disability Index; VAS, Visual Analog Scale; NRS, Numerical Rating Scale; SF-36, Short Form -36.

intermediate: $59 \%$ and late: $47.9 \%$. Furthermore, the distribution of the placebo effect among the various domains was significant for the four PROMs instruments $\left(X^{2}=78.08\right) \quad(\mathrm{P}<0.0001)$, the six anatomical targets $\left(X^{2}=910\right) \quad(\mathrm{P}=0)$ and the three follow up periods $\left(X^{2}=16.2\right)(\mathrm{P}=0.0003)$ (Table 4$)$.

\section{Discussion}

The use of sham procedures as part of clinical practice is dishonest, improper and unacceptable. Surgery involves trust and the close contact with the patient makes placebo effect more likely. ${ }^{2}$ Sham procedures are appropriate in the context of trials when the research in question is clinically important, the risks are acceptable and there is no deception. ${ }^{6,26}$ The sham arm allows for baseline comparison for the active intervention. ${ }^{26}$ Recent reviews demonstrated that the difference in the outcome between the sham and treated arms can be small implying that some procedures may be ineffective. ${ }^{1,27}$ A review of the nonoperative treatments for discogenic back pain concluded that it was unclear whether these interventions gave a stable long-term benefit. ${ }^{28}$ Furthermore, in this study, 9 out of the 17 reviewed trials reported comparable postprocedure PROMs between the sham and treated groups (Table 1). Hence, the use sham interventions to determine the efficacy of invasive procedures in back pain is clearly justified. $^{27}$

In study a placebo effect (significant reduction in follow up pain scores) was observed in $53.2 \%$ patients (and $21 / 55$ episodes) during the 6 months after sham spine procedures. The results are the within the wide range of $22-91 \%$ reported for pain improvement following sham surgical procedures. ${ }^{1,29}$ It is known that the magnitude of the placebo effect represents a compound product, only a portion of which may be attributable to the true placebo effect. $^{4}$ The remainder may reflect non-specific effects such as statistical factors, biological properties of the disease progression and psychological aspects of receiving attention by clinical staff. ${ }^{4,30}$ The placebo effect could be influenced by features such as the characteristics of the 
Table 4 The Distribution of the Pooled Number of Patients and Episodes That Had Significant and Non-Significant Reduction in Pain Scores Between the Various Domains of the PROMs, Anatomical Targets and Follow Up Periods

\begin{tabular}{|c|c|c|c|c|c|c|c|c|c|}
\hline \multirow[t]{2}{*}{ Parameters } & \multirow[t]{2}{*}{ Domains } & \multirow[t]{2}{*}{$\begin{array}{l}\text { Total } \\
\text { Studies }\end{array}$} & \multirow[t]{2}{*}{$\begin{array}{l}\text { Total } \\
\text { Episodes }\end{array}$} & \multicolumn{2}{|c|}{$\begin{array}{l}\text { Reduction in Pain } \\
\text { Scores By } \\
\text { Episodes Number }\end{array}$} & \multirow[t]{2}{*}{$\begin{array}{l}\text { Total } \\
\text { Patients }\end{array}$} & \multicolumn{3}{|c|}{$\begin{array}{l}\text { Reduction in Pain Scores By Patients } \\
\text { Number (\%) }\end{array}$} \\
\hline & & & & Sig. & Non-Sig. & & Sig. & Non-Sig. & $\begin{array}{l}X^{2} \\
\text { (P-value) }\end{array}$ \\
\hline PROMs instrument & $\begin{array}{l}\text { ODI } \\
\text { VAS } \\
\text { NRS } \\
\text { SF-36 }\end{array}$ & $\begin{array}{l}12 \\
6 \\
8 \\
6\end{array}$ & $\begin{array}{l}23 \\
12 \\
13 \\
7\end{array}$ & $\begin{array}{l}7 \\
4 \\
8 \\
2\end{array}$ & $\begin{array}{l}16 \\
8 \\
5 \\
5\end{array}$ & $\begin{array}{l}703 \\
389 \\
398 \\
151\end{array}$ & $\begin{array}{l}328(46.7 \%) \\
194(49.9 \%) \\
287(72.1 \%) \\
64(42.4 \%)\end{array}$ & $\begin{array}{l}375 \text { (53.3\%) } \\
195(50.1 \%) \\
I I I(27.9 \%) \\
87(57.6 \%)\end{array}$ & $\begin{array}{l}X^{2}=78.08 \\
(P<0.00 I)\end{array}$ \\
\hline $\begin{array}{l}\text { Anatomical target of } \\
\text { sham procedure }\end{array}$ & $\begin{array}{l}\text { Disc } \\
\text { Facet } \\
\text { BVN } \\
\text { RCN } \\
\text { SIJ } \\
\text { Caudal }\end{array}$ & $\begin{array}{l}6 \\
4 \\
1 \\
2 \\
3 \\
1\end{array}$ & $\begin{array}{l}23 \\
10 \\
4 \\
5 \\
9 \\
4\end{array}$ & $\begin{array}{l}2 \\
6 \\
4 \\
3 \\
2 \\
4\end{array}$ & $\begin{array}{l}21 \\
4 \\
0 \\
2 \\
7 \\
0\end{array}$ & $\begin{array}{l}542 \\
287 \\
308 \\
104 \\
160 \\
240\end{array}$ & $\begin{array}{l}60(11.1 \%) \\
151(52.6 \%) \\
308(100 \%) \\
67(64.4 \%) \\
47(29.4 \%) \\
240(100 \%)\end{array}$ & $\begin{array}{l}482(88.9 \%) \\
136(47.4 \%) \\
0 \\
37(35.6 \%) \\
113(70.6 \%) \\
0\end{array}$ & $\begin{array}{l}X^{2}=910 \\
(P=0)\end{array}$ \\
\hline $\begin{array}{l}\text { Follow up period in } \\
\text { months }\end{array}$ & $\begin{array}{l}\text { Early } \\
\text { Interm } \\
\text { Late }\end{array}$ & $\begin{array}{l}8 \\
9 \\
9\end{array}$ & $\begin{array}{l}14 \\
20 \\
21\end{array}$ & $\begin{array}{l}7 \\
8 \\
6\end{array}$ & $\begin{array}{l}7 \\
12 \\
15\end{array}$ & $\begin{array}{l}337 \\
648 \\
656\end{array}$ & $\begin{array}{l}177(52.5 \%) \\
382(59 \%) \\
314(47.9 \%)\end{array}$ & $\begin{array}{l}160(47.5 \%) \\
266(41 \%) \\
342(52.1 \%)\end{array}$ & $\begin{array}{l}X^{2}=16.2 \\
(P<0.001)\end{array}$ \\
\hline
\end{tabular}

Notes: Early, 0-2 months; intermediate, >2-4 months; late, >4-6 month.

Abbreviations: Sig, significant; RROMS, Patient Reported Outcome Measures; Interm, intermediate; BVN, basivertebral nerve; RCN, Ramus Communicans Nerve; SIJ, sacroiliac joint; $X^{2}$, chi-squared; ODI, Oswestry Disability Index; VAS, Visual Analog Scale; NRS, Numerical Rating Scale; SF-36, Short Form -36.

treatment, the healthcare-setting, the clinician, the patient,

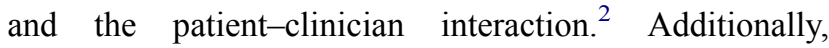
genetic, geographical, and cultural factors may be relevant. ${ }^{2}$ The psychological and neurobiological mechanisms underlying the placebo effect are dominated by two main theories. The first is the associative learning theory in which pairing of the placebo with the active treatment triggers a physiological response. ${ }^{2}$ The second is the response expectancy theory in which the patient's conscious or unconscious expectation that the placebo will have a particular effect. ${ }^{2,31}$ Some researchers believe that the patient expectations are the central psychological mechanism. ${ }^{2,31}$ Expectations trigger a cascade of endogenous opioids and non-opioids which alter the experience of pain. $^{30}$

The management of low back pain is complicated by the myriads of PROMs used to assess the response to treatment. ${ }^{32}$ It is agreed that the various instruments differ in their domain-focus and several reviews recommended the use of ODI and SF-36 for physical functioning, ${ }^{32,33}$ and NRS and VAS for pain. ${ }^{32-34}$ In this study we observed a significant difference in the placebo effect according to the utilized PROMs instruments. The highest effect was seen using NRS (72.1\% patients and in 8/13 episodes) while the usage of ODI, VAS, and SF-36 was associated with a placebo effect in $46.7 \%, 49.9 \%$ and $42.4 \%$ of patients, respectively. These findings are not unusual as pain scoring was the focus of the study. NRS is recognized as the preferred measure for pain intensity in back pain and while it has good sensitivity it generates data that can be analyzed for audit purposes. ${ }^{34}$ VAS is also appropriate for pain scoring however, a few studies cited difficulties in its use by the elderly and populations with disadvantages. $^{34}$

A significant difference in the placebo effect was observed based on the anatomical target for the sham procedure. The lowest effect was seen with the disc $(11.1 \%$ patients and 2/23 episodes). Targeting the disc was associated with no significant difference between the treated and the sham arms in four of the selected trials, ${ }^{12,16-18}$ and only a partial improvement in two trials ${ }^{10,21}$ (Table 1). A relatively low placebo effect was noted with the targeting of SIJ (29.4\% patients and 2/9 episodes). However, sham spine procedures that targeted the facets, RCN, BVN and caudal were associated with relatively better placebo effect. Some of the differences are the result of bias due the small numbers of episodes and patients. The variation however could be related to the quantity of information and the 
manner the patients were informed about the benefit of the procedure and their level of expectations. It is known the cognitive educational approach of explaining pain and pain processing to patients could alter their perception of pain. ${ }^{5}$ It is also recognized that an imbalance between the quantity of information given about the benefits of the procedure could influence the response. ${ }^{2}$

There is no consensus on the influence of time on the placebo effect. Some studies reported that pain and subjective outcomes of the placebo effect are not affected by time. ${ }^{4,29}$ Others observed the largest improvement in outcome during the $0-1$ month interval. ${ }^{1}$ We observed a difference in the significant reduction in pain scores between the three follow up periods. The placebo effect was higher in the $0-2$ months period $(52.5 \%$ patients and $7 / 14$ episodes) and the $>2-4$ months period (59\% patients and $8 / 20$ episodes) compared to the $>4-6$ months period (47.9\% patients and 6/21 episodes). Our findings support the view that placebo effect following sham procedures has time effect curve with a height and a carryover effect, that slowly declines with time. ${ }^{4}$

There are several limitations to this study. There may have been some missing trials. The studies were broadly heterogenous with regards to the nature of the treatment modality, the method of pain assessment and the follow up time points. There was also diversity in the number of patients and follow up pain scoring episodes in the various articles. The influence of the injection of LA, the number of face-to-face visits, the duration of the sham procedure were not assessed. Furthermore, the matter of presence or absence of concomitant treatment was not addressed in the selected trials. One plausible explanation for the improvement could have been the use of medication or lifestyle modification.

\section{Conclusions}

Ethical consideration dictates limiting the use of sham procedures except in well indicated clinical trials. At a follow up of 6 months, a placebo effect was experienced by nearly half of the patients who underwent sham spine procedures. The placebo effect was significantly influenced by the utilized pain scoring instrument, the anatomical target for the sham procedure and the duration of the follow up. Further research is required to explore the extent to which the observed improvement in the follow up pain scores was due true placebo effect and not other sources of bias. The findings should be taken into consideration in the design of new sham-controlled spine procedure trials particularly in the selection of PROMs, the choice of the target for the procedure and the length of follow up.

\section{Ethics Approval and Consent to Participate}

No ethical approval was necessary as the study was based on data obtained from open access sources.

\section{Author Contributions}

All authors contributed to data analysis, drafting or revising the article, have agreed on the journal to which the article will be submitted, gave final approval of the version to be published, and agree to be accountable for all aspects of the work.

\section{Funding}

No funding was received.

\section{Disclosure}

The authors declare that they have no competing interests.

\section{References}

1. Gu AP, Gu CN, Ahmed AT, et al. Sham surgical procedures for pain intervention result in significant improvements in pain: systematic review and meta-analysis. $J$ Clin Epidemiol. 2017;83:18-23. doi:10.1016/j.jclinepi.2016.12.010

2. Beard DJ, Campbell MK, Blazeby JM, et al. Considerations and methods for placebo controls in surgical trials (ASPIRE guidelines). Lancet. 2020;395(10226):828-838. doi:10.1016/S0140-6736(19) 33137-X

3. Cousins S, Blencowe NS, Blazeby JM. What is an invasive procedure? A definition to inform study design, evidence synthesis and research tracking. BMJ Open. 2019;9(7):e028576. doi:10.1136/bmjopen-2018-028576

4. Wartolowska KA, Feakins BG, Collins GS, et al. The magnitude and temporal changes of response in the placebo arm of surgical randomized controlled trials: a systematic review and meta-analysis. Trials. 2016;17(1):589. doi:10.1186/s13063-016-1720-7

5. Louw A, Diener I, Fernandez-de-las-penas C, Puentedura EJ. Sham surgery in orthopaedics: a systematic review of the literature. Pain Med. 2017;18:736-750. doi:10.1093/pm/pnw164

6. Savulescu J, Wartolowska K, Carr A. Randomised placebo-controlled trials of surgery: ethical analysis and guidelines. J Med Ethics. 2016;42(12):776-783. doi:10.1136/medethics-2015-103333

7. MedCalc statistical software. Belgium: MedCalc Software Ltd. Available from: https://www.medcalc.org. Accessed January 15, 2021.

8. Mathsisfun statistical software. Available from: https://www.mathsis fun.com. Accessed January 15, 2021.

9. Leclaire R, Fortin L, Lambert R, Bergeron YM, Rossignol M. Radiofrequency facet joint denervation in the treatment of low back pain: a placebo-controlled clinical trial to assess efficacy. Spine. 2001;26(13):1411-1416. doi:10.1097/00007632-20010701000003

10. Pauza KJ, Howell S, Dreyfuss P, Peloza JH, Dawson K, Bogduk N. A randomized, placebo-controlled trial of intradiscal electrothermal therapy for the treatment of discogenic low back pain. Spine $J$. 2004;4(1):27-35. doi:10.1016/j.spinee.2003.07.001 
11. Oh WS, Shim JC. A randomized controlled trial of radiofrequency denervation of the ramus communicans nerve for chronic discogenic low back pain. Clin J Pain. 2004;20:55-60. doi:10.1097/00002508200401000-00011

12. Freeman BJ, Fraser RD, Cain CM, Hall DJ, Chapple DC. A randomized, double-blind, controlled trial: intradiscal electrothermal therapy versus placebo for the treatment of chronic discogenic low back pain. Spine. 2005;30(21):2369-2377. doi:10.1097/01. brs.0000186587.43373.f2

13. van Wijk RM, Geurts JW, Wynne HJ, et al. Radiofrequency denervation of lumbar facet joints in the treatment of chronic low back pain: a randomized, double-blind, sham lesion-controlled trial. Clin J Pain. 2005;21(4):335-344. doi:10.1097/01.ajp.0000120792.69705.c9

14. Tekin I, Mirzai H, Ok G, et al. A comparison of conventional and pulsed radiofrequency in denervation in the treatment of chronic facet joint pain. Clin J Pain. 2007;23:524. doi:10.1097/AJP.0b $013 \mathrm{e} 318074 \mathrm{c} 99 \mathrm{c}$

15. Cohen SP, Hurley RW, Buckenmaier CC, et al. Randomized placebo-controlled study evaluating lateral branch radiofrequency denervation for sacroiliac pain. Anesthesiology. 2008;109:279-288. doi:10.1097/ALN.0b013e31817f4c7c

16. Kvarstein G, Måwe L, Indahl A, et al. A randomized double-blind controlled trial of intra-annular radiofrequency thermal disc therapya 12-month follow-up. Pain. 2009;145(3):279-286. doi:10.1016/j. pain.2009.05.001

17. Peng B, Pang X, Wu Y, Zhao C, Song A. A randomized placebo-controlled trial of intradiscal methylene blue injection for the treatment of chronic discogenic low back pain. Pain. 2010;149 (1):124-129. doi:10.1016/j.pain.2010.01.021

18. Cao $\mathrm{P}$, Jiang $\mathrm{L}$, Zhuang $\mathrm{C}$, et al. Intradiscal injection therapy for degenerative chronic discogenic low back pain with end plate Modic changes. Spine J. 2011;11(2):100-106. doi:10.1016/j.spinee.20 10.07.001

19. Patel N, Gross A, Brown L, et al. A randomized placebo-controlled study to assess the efficacy of lateral branch neurotomy for chronic sacroiliac joint pain. Pain Med. 2012;13:383-398. doi:10.1111/ j.1526-4637.2012.01328.x

20. Manchikanti L, Cash KA, McManus CD, Pampati V. Fluoroscopic caudal epidural injections in managing chronic low back pain without disc herniation radiculitis or facet joint pain. $J$ Pain Res. 2012;5:381-390. doi:10.2147/JPR.S35924

21. Kapural L, Vrooman B, Sarwar S, et al. A randomized, placebo-controlled trial of transdiscal radiofrequency, biacuplasty for treatment of discogenic lower back pain. Pain Med. 2013;14 (3):362-373. doi:10.1111/pme.12023

22. van Tilburg CW, Stronks DL, Groeneweg JG, Huygen FJ. Randomised sham-controlled double-blind multicentre clinical trial to ascertain the effect of percutaneous radiofrequency treatment for lumbar facet joint pain. Bone Joint J. 2016;98- B(11):1526-1533. doi:10.1302/0301-620X.98B11.BJJ-2016-0379.R2
23. van Tilburg CW, Schuurmans FA, Stronks DL, Groeneweg JG, Huygen FJ. Randomized sham-controlled double-blind multicenter clinical trial to ascertain the effect of percutaneous radiofrequency treatment for sacroiliac joint pain: three-month results. Clin J Pain. 2016;32(11):921-926. doi:10.1097/AJP.0000000000000351

24. van Tilburg CW, Stronks DL, Groeneweg JG, Huygen FJ. Randomized sham-controlled, double-blind, multicenter clinical trial on the effect of percutaneous radiofrequency at the ramus communicans for lumbar disc pain. Eur J Pain. 2017;21(3):520-529. doi:10.1002/ejp.945

25. Fischgrund JS, Rhyne A, Franke J, et al. Intraosseous basivertebral nerve ablation for the treatment of chronic low back pain: a prospective randomized double-blind sham-controlled multi-center study. Eur Spine J. 2018;27(5):1146-1156. doi:10.1007/s00586-0185496-1

26. Hostiuc S, Rentea I, Drima E, Negoi I. Placebo in surgical research: a case-based ethical analysis and practical consequences. Biomed Res Int. 2016:2627181. doi:10.1155/2016/2627181

27. Jonas WB, Crawford C, Colloca L, et al. To what extent are surgery and invasive procedures effective beyond a placebo response? A systematic review with meta-analysis of randomized, sham controlled trials. BMJ Open. 2015;5(12):e009655. doi:10.1136/bmjopen2015-009655

28. Lu Y, Guzman JZ, Purmessur D, et al. Nonoperative management of discogenic back pain: a systematic review. Spine. 2014;39 (16):1314-1324. doi:10.1097/BRS.0000000000000401

29. Wartolowska KA, Gerry S, Feakins BG, et al. A meta-analysis of temporal changes of response in the placebo arm of surgical randomized controlled trials: an update. Trials. 2017;18(1):1-7. doi:10.1186/s13063-017-2070-9

30. Klinger R, Stuhlreyer J, Schwartz M, Schmitz J, Colloca L. Clinical use of placebo effects in patients with pain disorders. Int Rev Neurobiol. 2018;139:107-128. doi:10.1016/bs.irn.2018.07.015

31. Vase L, Wartolowska K. Pain, placebo, and test of treatment efficacy: a narrative review. $B r \quad J$ Anaesth. 2019;123(2):e254-e262. doi:10.1016/j.bja.2019.01.040

32. Chapman JR, Norvell DC, Hermsmeyer JT, et al. Evaluating common outcomes for measuring treatment success for chronic low back pain. Spine. 2011;36(21 Suppl):S54-S68. doi:10.1097/BRS.0b013e 31822ef74d

33. Chiarotto A, Boers M, Deyo RA, et al. Core outcome measurement instruments for clinical trials in nonspecific low back pain. Pain. 2018;159(3):481-495. doi:10.1097/j.pain.0000000000001117

34. Karcioglu O, Topacoglu H, Dikme O, Dikme O. A systematic review of the pain scales in adults: which to use? Am J Emerg Med. 2018;36 (4):707-714. doi:10.1016/j.ajem.2018.01.008
Journal of Pain Research

\section{Publish your work in this journal}

The Journal of Pain Research is an international, peer reviewed, open access, online journal that welcomes laboratory and clinical findings in the fields of pain research and the prevention and management of pain. Original research, reviews, symposium reports, hypothesis formation and commentaries are all considered for publication. The manuscript

Submit your manuscript here: https://www.dovepress.com/journal-of-pain-research-journal management system is completely online and includes a very quick and fair peer-review system, which is all easy to use. Visit http:// www.dovepress.com/testimonials.php to read real quotes from published authors. 